\title{
A Spline-Trigonometric Galerkin Method and an Exponentially Convergent Boundary Integral Method
}

\author{
By Douglas N. Arnold*
}

\begin{abstract}
We consider a Galerkin method for functional equations in one space variable which uses periodic cardinal splines as trial functions and trigonometric polynomials as test functions.

We analyze the method applied to the integral equation of the first kind arising from a single layer potential formulation of the Dirichlet problem in the interior or exterior of an analytic plane curve. In constrast to ordinary spline Galerkin methods, we show that the method is stable, and so provides quasioptimal approximation, in a large family of Hilbert spaces including all the Sobolev spaces of negative order. As a consequence we prove that the approximate solution to the Dirichlet problem and all its derivatives converge pointwise with exponential rate.
\end{abstract}

1. Introduction. In this paper we formulate and analyze a new method for the numerical solution of functional equations such as integral and differential equations. Our method is particularly appropriate for the solution of integral equations arising from boundary integral formulations of boundary value problems in partial differential equations. The desired solution to the boundary value problem is typically obained at an interior point of the domain by integrating the solution of the boundary integral equation times a smooth weighting function. In conjunction with this procedure our method of solution of the integral equation will lead to exponentially fast convergence of the solution and its derivatives away from the boundary, in contrast to the polynomial rates of convergence achieved by the majority of other practical discretization methods, such as finite element, finite difference, and collocation methods.

The method we propose for solving the integral equation is a Galerkin method with spline trial functions and trigonometric test functions, which we shall refer to as the spline-trig method. Spline Galerkin methods, or finite element methods, in which both the test and trial spaces consist of splines, have been widely studied. It is however the novel aspect of the spline-trig method, the use of trigonometric polynomials as test functions, which leads to the exponential convergence rates referred to above.

Because of the novelty of the method and its analysis we shall not strive for generality in this paper, but instead shall study the method in the context of one relatively simple model problem. The problem we consider is the (interior and

Received October 5, 1982; revised February 1, 1983.

1980 Mathematics Subject Classification. Primary 65R20; Secondary 65N30, 65E05, 45L10, 45B05, $41 \mathrm{~A} 15$.

* This research was supported by the National Science Foundation under Grant MCS-8102012 and was completed while the author was a NATO Postdoctoral Fellow at the Technische Hochschule Darmstadt. 
exterior) Dirichlet problem for Laplace's equation in two dimensions, to be solved using a single layer potential formulation. Let $\Gamma$ be a smooth simple closed curve in the plane and let $\tilde{g}$ be a given function on $\Gamma$. We seek a bounded continuous function $u$ on $\mathbf{R}^{2}$ such that

$$
\begin{aligned}
\Delta u=0 & & \text { on } \mathbf{R}^{2} \backslash \Gamma, \\
u=\tilde{g} & & \text { on } \Gamma .
\end{aligned}
$$

We shall represent the solution of (1.1) as a single layer potential. In order to avoid difficulties of nonuniqueness when the interior domain has conformal radius one, we follow [6], [11], [14] in seeking to express $u$ as a normalized single layer potential plus a constant. That is, we seek a function $\psi$ on $\Gamma$ of mean value zero and a real number $\omega$ such that

$$
u(z)=-\frac{1}{2 \pi} \int_{\Gamma} \log |z-y| \psi(y) d s_{y}+\omega, \quad z \in \mathbf{R}^{2} .
$$

Restricting $z$ to $\Gamma$, we get the integral equation

$$
-\frac{1}{2 \pi} \int_{\Gamma} \log |z-y| \psi(y) d s_{y}+\omega=\tilde{g}(z), \quad z \in \Gamma .
$$

This equation has a unique solution. (In Section 4 we show, more precisely, that the operator $(\psi, \omega) \mapsto \tilde{g}$ defined by (1.3) is an isomorphism $\hat{H}^{r}(\Gamma) \times \mathbf{R} \rightarrow H^{r+1}(\Gamma)$ for any real number $r$. The space $H^{r}(\Gamma)$ is the Sobolev space of order $r$ on $\Gamma$, which is precisely defined in Section 3. The circumflex denotes the subspace of codimension one consisting of elements of mean value zero.) The unique solution $u$ of (1.1) is given by (1.2). For a proof of these facts see [9], [11], [14]. We remark that with the single layer approach the solution to the single integral equation (1.3) enables the determination of the solution to both the interior and exterior problems.

In Section 2 of this paper we define and discuss the spline-trig method for our problem. In Section 3 we define a special doubly indexed family of Hilbert spaces and establish some properties we will require in the analysis of the method, which is accomplished in the rest of the paper. In Section 4 it is shown that the method provides stable, quasioptimal approximation of the solution of the integral equation in a wide range of spaces including all the Sobolev spaces of negative order. The rates of convergence in these spaces is determined in Section 5, and it is shown that the corresponding approximation to the solution of the Dirichlet problem converges along with all its derivatives with an exponential rate away from the boundary. Polynomial convergence rates valid up to the boundary are also proved. In the final section it is shown that the matrices arising from the discretization are well-conditioned.

2. The Spline-Trig Galerkin Method. Rather than approximate the solution of (1.3) directly, it is more convenient to parametrize $\Gamma$ and change variables. We assume that $\Gamma$ admits an analytic parametrization, i.e., that we can find a real analytic, 1-periodic function $x: \mathbf{R} \rightarrow \mathbf{R}^{2}$ such that $x^{\prime}$ does not vanish and $\left.x\right|_{[0,1]}$ parametrizes $\Gamma$. (For a discussion of the effect of weakening the analyticity condition, see Remarks 4.11 and 5.6.) Set $g(t)=\tilde{g}(x(t))$ and

$$
\varphi(t)=\frac{1}{2} \psi(x(t))\left|x^{\prime}(t)\right|+\omega .
$$


We use the notation $\hat{\varphi}(0)$ for $\int_{0}^{1} \varphi(t) d t$. Note that $\omega$ may be recovered from $\varphi$ as $\hat{\varphi}(0)$, and then $\psi$ may be uniquely determined from $\varphi$ and satisfies $\int_{\Gamma} \psi=0$. Thus (2.1) establishes an isomorphism $(\psi, \omega) \leftrightarrow \varphi$ (between the spaces $\hat{H}^{r}(\Gamma) \times \mathbf{R}$ and $H^{r}$, $r \in \mathbf{R}$, defined in Section 3).

In terms of the new unknown, $\varphi,(1.2)$ and (1.3) become

$$
\begin{aligned}
& u(z)=-\frac{1}{\pi} \int_{0}^{1} \log \mid z-x(t) \|[\varphi(t)-\hat{\varphi}(0)] d t+\hat{\varphi}(0), \quad z \in \mathbf{R}^{2}, \\
& A \varphi(\tau):=-\frac{1}{\pi} \int_{0}^{1} \log \mid x(\tau)-x(t) \|[\varphi(t)-\hat{\varphi}(0)] d t+\hat{\varphi}(0)=g(\tau), \\
& \tau \in \mathbf{R} .
\end{aligned}
$$

We shall study the spline-trig method for the integral equation (2.3). This is a Galerkin method employing different test and trial spaces. It is novel in that spline trial functions are used-as in finite element methods-but trigonometric test functions are used - as in spectral methods.

Let $n$ and $d$ henceforth denote nonnegative integers, with $n$ odd. Let

$$
\Lambda_{n}=\{p \in \mathbf{Z}|| p \mid<n / 2\},
$$

a set of representatives for $\mathbf{Z}$ modulo $n$, and let $\chi_{n}^{1}$ denote $n$ times the characteristic function of $\cup\{[m-1 / 2 n, m+1 / 2 n] \mid m \in \mathbf{Z}\}$. For $d>0$ define $\chi_{n}^{d+1}$ as the convolution

$$
\chi_{n}^{d+1}(x)=\int_{0}^{1} \chi_{n}^{d}(x-y) \chi_{n}^{1}(y) d y .
$$

As trial space we will use the space $\Im_{n}^{d}$ spanned by all translates of $\chi_{n}^{d+1}$ by integral multiples of $1 / n$. This space has dimension $n$ and consists of all 1 -periodic smoothest splines of degree $d$ subordinate to the uniform mesh $\{j / n \mid j \in \mathbf{Z}\}$ if $d$ is odd, $\{(j+1 / 2) / n \mid j \in \mathbf{Z}\}$ if $d$ is even [15], [16]. The $B$-splines $\chi_{n}^{d+1}(\cdot-j / n)$, $j \in \Lambda_{n}$, form a basis for $\varsigma_{n}^{d}$. It is a local basis as each $B$-spline is supported in $d+1$ consecutive mesh subintervals and their 1-periodic translates.

As test space we shall use the span, $\mathcal{T}_{n}$, of the trigonometric monomials $1, \sin 2 \pi x$, $\cos 2 \pi x, \sin 4 \pi x, \ldots, \cos (n-1) \pi x$.

The spline-trig method for the equation (2.3) defines $\varphi_{n} \in \mathcal{S}_{n}^{d}$ by the conditions

$$
\int_{0}^{1} A \varphi_{n} \sigma=\int_{0}^{1} g \sigma, \quad \sigma \in \mathscr{T}_{n} .
$$

(Since we are concerned with the asymptotic behavior of $\varphi_{n}$ as $n \rightarrow \infty$ with $d$ fixed, we shall not indicate the dependence of $\varphi_{n}$ on $d$ in the notation.) The coefficients of $\varphi_{n}$ with respect to the $B$-splines or other basis of $\delta_{n}^{d}$ may be determined as the solution to an $n \times n$ system of linear equations determined by (2.4).

The fact that the space $\mathcal{T}_{n}$ does not 2 dmit a local basis is possibly a disadvantage of the present method in comparison with ordinary finite element methods. As a consequence, the matrix elements entering into the linear system corresponding to (2.4) must be calculated as double integrals in which one integral is over all of $[0,1]$ rather than just a small number of mesh subintervals. On the other hand the use of trigonometric trial functions in the method suggests that the matrix columns can be calculated quickly with aid of the fast Fourier transform. Note that the matrix is not 
sparse, but this would be so even if a test space with a local basis were used, due to the nonlocal nature of the operator $A$. A full analysis of the implementation costs of the spline-trig method and a comparison with other methods must await further research on suitable techniques of quadrature of the singular integrands (ideas from [10] may be useful here) and the effects of quadrature errors on the accuracy of the method, as well as numerical experimentation.

Finite element methods are usually analyzed in the Sobolev spaces. Typically a method is stable (i.e., the Galerkin projection is bounded independent of the meshsize) in a Sobolev space of only one particular order or a certain range of orders. (E.g., standard finite element methods for second order elliptic problems are stable in the Sobolev space of order one.) This stability implies (in fact is equivalent to) the quasioptimality of the method, i.e., that the norm of the error in the space or spaces for which stability holds is bounded by a constant times the distance of the solution from the trial space measured in the same norm. For a bounded range of lower order Sobolev spaces it is also generally possible to prove that the asymptotic rate of convergence is optimal. Now in many situations it can be seen that the least Sobolev order for which such optimal order convergence holds is tied to the degree of the splines which are used as test functions [1], [2], [17, Section 2.3]. The greater the degree of the test functions, the farther down extends the range of spaces in which optimal order approximation holds. Now in a certain sense the space $\widetilde{T}_{n}$ of trigonometric polynomials may be viewed as the limit of the spline spaces $\varsigma_{n}^{d}$ as $d \rightarrow \infty$. In fact using the Fourier characterization of splines (4.6) it is not difficult to prove that

$$
\begin{aligned}
\mathscr{T}_{n} & =\left\{f \in L^{2} \mid \exists s_{d} \in S_{n}^{d}, d \in \mathbf{N}, \text { such that } s_{d} \stackrel{L^{2}}{\rightarrow} f \text { as } d \rightarrow \infty\right\} \\
& =\left\{f \in L^{2} \mid \exists d_{k} \in \mathbf{N} \text { and } s_{k} \in \mathcal{S}_{n}^{d_{k}}, \text { such that } d_{k} \rightarrow \infty, s_{k} \stackrel{L^{2}}{\rightarrow} f \text { as } k \rightarrow \infty\right\} .
\end{aligned}
$$

These observations motivate the use of trigonometric test functions and suggest that our method might achieve optimal order approximation in an unbounded range of Sobolev spaces. In fact we shall show that the spline-trig method even achieves stable (so quasioptimal) approximation in all Sobolev spaces of negative order. Once such approximation is demonstrated, it will follow easily that for any $z \in \mathbf{R}^{2} \backslash \Gamma$ the approximation to $u(z)$ determined by substituting $\varphi_{n}$ for $\varphi$ in (2.2) will converge faster than any power of $1 / n$. We will prove an even stronger result, exponential convergence to $u(z)$ and its derivatives, by introducing a family of spaces which are weaker than the Sobolev spaces and establishing stability in these spaces.

3. A Family of Hilbert Spaces. Let $\mathcal{T}=\cup\left\{\mathscr{T}_{n} \mid n \in \mathbf{N}\right\}$ denote the space of trigonometric polynomials. We write an arbitrary element of $\mathcal{T}$ as

$$
f(t)=\sum_{k \in \mathbf{Z}} \hat{f}(k) e^{2 \pi i k t},
$$

where the $\hat{f}(k)$ are arbitrary complex numbers, all but finitely many zero, satisfying $\hat{f}(k)=\overline{\hat{f}(-k)}$. We henceforth shall use the notation $\underline{k}=\max (2 \pi|k|, 1)$ for $k \in \mathbf{Z}$. Now for each $\varepsilon, s \in \mathbf{R}, \varepsilon>0$, and $f \in \mathcal{T}$ given by (3.1) define

$$
\|f\|_{s, \varepsilon}=\left[\sum_{k}|\hat{f}(k)|^{2} \varepsilon^{2|k|} \underline{k}^{2 s}\right]^{1 / 2} .
$$


(In this paper summation indices are assumed to vary over all integers unless otherwise indicated.) The Hilbert space $X_{s, \varepsilon}$ is defined as the completion of $\mathcal{T}$ in this norm. Clearly we have continuous dense injections (which we may take to be inclusions),

$$
X_{s_{1}, \varepsilon_{1}} \subset X_{s_{2}, \varepsilon_{2}},
$$

if either $\varepsilon_{1}>\varepsilon_{2}$ or $\varepsilon_{1}=\varepsilon_{2}$ and $s_{1}>s_{2}$. The spaces $H^{s}:=X_{s, 1}$ are the periodic Sobolev spaces [1], their elements being 1-periodic distributions. We will use the usual notation $\|\cdot\|_{s}$ in place of $\|\cdot\|_{s, 1}$ for the $H^{s}$ norm. (The space $H^{r}(\Gamma)$ referred to in Sections 1 and 2 may now be defined as the set of functions on $\Gamma$ whose composition with the parametrization $x$ lies in $H^{r}$, with norm being the $H^{r}$ norm of this composition.) The space $H^{0}$ is identical with $L^{2}$, the space of 1-periodic measurable functions which are square integrable over a period. If $\varepsilon>1$, the elements of $X_{s, \varepsilon}$ are infinitely differentiable functions. However, for $\varepsilon<1$, the elements of $X_{s, \varepsilon}$ need not be distributions. We remark that the $X_{s, \varepsilon}$ norm may also be defined by

$$
\|f\|_{s, \varepsilon}=\left\|T\left(\frac{1}{2 \pi} \log \varepsilon\right) f\right\|_{s}
$$

where $T$ is the semigroup generated by the positive definite square root of the operator $-d^{2} / d x^{2}$. Norms of this form are used very effectively in [7] which inspired their use in this analysis.

By density there exists for $k \in \mathbf{Z}$ a unique extension of the linear functional on $\mathcal{T}$

$$
f \mapsto \hat{f}(k)=\int_{0}^{1} f(t) e^{-2 \pi i k t} d t, \quad k \in \mathbf{Z},
$$

to a continuous linear functional on $X_{s, \varepsilon}, s \in \mathbf{R}, \varepsilon>0$. Moreover, these extensions are compatible for different $s$ and $\varepsilon$, i.e., they are each the restriction of a single linear function on the vectorspace

$$
X:=\bigcup\left\{X_{s, \varepsilon} \mid s \in \mathbf{R}, \varepsilon>0\right\} .
$$

Define

$$
\|f\|_{s, \varepsilon}=+\infty, \quad f \in X \backslash X_{s, \varepsilon} .
$$

With these understandings the equation (3.2) is valid for all $f \in X, s \in \mathbf{R}, \varepsilon>0$.

The $L^{2}$ innerproduct,

$$
(f, g):=\int_{0}^{1} f g=\sum \hat{f}(k) \overline{\hat{g}(k)},
$$

extends to a real-valued bilinear map on $X_{s, \varepsilon} \times X_{-s, \varepsilon^{-1}}$ for all $s \in \mathbf{R}, \varepsilon>0$. These extensions are all compatible and provide a canonical isomorphism of $X_{s, \varepsilon}$ with the dual space of $X_{-s, \varepsilon^{-1}}$.

It is easily checked that a subset $\mathcal{S}$ of $X_{s, \varepsilon}$ is precompact if and only if it is bounded in $X_{s, \varepsilon}$ and

$$
\lim _{m \rightarrow \infty} \sup _{f \in \delta} \sum_{|k| \geqslant m}|\hat{f}(k)|^{2} \varepsilon^{2|k|} \underline{k}^{2 s}=0 .
$$

It follows that the inclusions indicated in (3.3) are compact. 
4. Stability Analysis. For the remainder of this paper $d$ denotes a nonnegative integer, $n$ a positive odd integer.

Since the test and trial spaces in the spline-trig method are not simply related, it is not clear that the Galerkin projection $\varphi \mapsto \varphi_{n}$ defined by (2.4) is stable in any reasonable space. (If the test and trial spaces are equal, by way of contrast, stability in $H^{-1 / 2}$ follows immediately from the ellipticity of the pseudodifferential operator $A$ [2], [9], [12], [13], [14].) To establish stability we shall rely on Fourier analysis. We begin by decomposing the operator $A$ as a sum of an operator which acts very simply on Fourier coefficients and a compact operator.

Define

$$
V \varphi(\tau)=-\frac{1}{\pi} \int_{0}^{1}[\log |2 \sin \pi(\tau-t)|-\pi] \varphi(t) d t, \quad \varphi \in L^{2} .
$$

Note that since $\left|e^{2 \pi i \tau}-e^{2 \pi i t}\right|=|2 \sin \pi(\tau-t)|$ and

$$
\int_{0}^{1} \log |2 \sin \pi(\tau-t)| d t=0, \quad \tau \in \mathbf{R}
$$

$V$ coincides with $A$ in case $\Gamma$ is the unit circle and $x(\tau)=e^{2 \pi i \tau}$. We now analyze the mapping properties of $V$. Let $G$ denote the convolution kernel in (4.1),

$$
G(\theta)=-\frac{1}{\pi} \log |2 \sin \pi \theta|+1=-\frac{1}{\pi} \log \left|1-e^{2 \pi i \theta}\right|+1, \quad \theta \in \mathbf{R} .
$$

Now, letting Log: $\mathbf{C} \backslash\{t \leqslant 0\} \rightarrow \mathbf{C}$ denote the holomorphic extension of log: $\{t>0\}$

$\rightarrow \mathbf{R}$, we have for $r \in(0,1)$

$$
\begin{aligned}
-\sum_{k \in \mathbf{Z}^{*}} \frac{r^{|k|} e^{2 \pi i k \theta}}{2|k|} & =-\sum_{k=1}^{\infty} \frac{r^{k} \cos (2 \pi i k \theta)}{k} \\
& =-\operatorname{Re} \sum_{k=1}^{\infty} \frac{r^{k} e^{2 \pi i k \theta}}{k}=\operatorname{Re} \log \left(1-r e^{2 \pi i \theta}\right)=\log \left|1-r e^{2 \pi i \theta}\right| .
\end{aligned}
$$

(The asterisk appended to a set of integers indicates the complement of $\{0\}$ in that set.) This equality exhibits the Fourier series of $\theta \mapsto \log \left|1-r e^{2 \pi i \theta}\right|$. Letting $r$ increase to 1 , we see that $\hat{G}(k)=\underline{k}^{-1}, k \in \mathbf{Z}$. Consequently the convolution formula for Fourier series yields the well-known representation

$$
\widehat{V \rho}(k)=\hat{\rho}(k) \underline{k}^{-1}, \quad k \in \mathbf{Z}, \rho \in L^{2} .
$$

It follows immediately that $V$ has a unique extension to a linear map $X \rightarrow X$ which maps $X_{s, \varepsilon}$ isometrically onto $X_{s+1, \varepsilon}$ for every $s \in \mathbf{R}, \varepsilon>0$. Accordingly

$$
|(V \rho, \sigma)| \leqslant\|\rho\|_{s, \varepsilon}\|\boldsymbol{\sigma}\|_{-s-1, \varepsilon^{-1}}, \quad \rho, \sigma \in X, s \in \mathbf{R}, \varepsilon>0 .
$$

The next theorem establishes the stability of the spline-trig projection for the operator $V$.

THEOREM 4.1. Let $d_{0}$ be a nonnegative integer, $s_{0} \in\left(-\infty, d_{0}+1 / 2\right)$. Then there exists a positive constant $C_{1}$ such that

$$
\inf _{0 \neq \rho \in \mathcal{S}_{n}^{d}} \sup _{0 \neq \sigma \in \mathcal{T}_{n}} \frac{(V \rho, \sigma)}{\|\rho\|_{s, \varepsilon}\|\sigma\|_{-s-1, \varepsilon^{-1}}} \geqslant C_{1}
$$

for all $d \geqslant d_{0}, s \in\left(-\infty, s_{0}\right], \varepsilon \in(0,1]$, and $n$. 
The proof relies on the following bound for the $X_{s, \varepsilon}$ norm of a spline in terms of its first $n$ Fourier coefficients.

LEMMA 4.2. For each nonnegative integer $d_{0}$ and each $s_{0} \in\left(-\infty, d_{0}+1 / 2\right)$ there exists a positive constant $C_{2}$ such that

$$
\|\rho\|_{s, \varepsilon}^{2} \leqslant C_{2} \sum_{p \in \Lambda_{n}}|\hat{\rho}(p)|^{2} \varepsilon^{2|p|} \underline{p}^{2 s}, \quad \rho \in \varsigma_{n}^{d},
$$

for all $d \geqslant d_{0}, s \in\left(-\infty, s_{0}\right], \varepsilon \in(0,1]$, and $n$.

Proof of Lemma 4.2. We begin by recalling the Fourier series characterization of the spline spaces,

$$
\varsigma_{n}^{d}=\left\{\rho \in L^{2} \mid \hat{\rho}(m) m^{d+1}=\hat{\rho}(m+n)(-m-n)^{d+1}, m \in \mathbf{Z}\right\} .
$$

In fact, in case $\rho(x)=\chi_{n}^{d+1}(x-j / n), j \in \mathbf{Z}$, (where $\chi_{n}^{d+1}$ is the $B$-spline defined in Section 2), then

$$
\begin{aligned}
\hat{\rho}(m) m^{d+1} & =e^{-2 \pi i m j / n} \hat{\chi}_{n}^{d+1}(m) m^{d+1} \\
& =e^{-2 \pi i m j / n}\left[\hat{\chi}_{n}^{1}(m) m\right]^{d+1}=e^{-2 \pi i m j / n}\left[\sin (\pi m / n) n \pi^{-1}\right]^{d+1} .
\end{aligned}
$$

The inclusion of $\varsigma_{n}^{d}$ in the space asserted in (4.6) follows easily. Equality holds since this space has dimension $n$ : the values $\hat{\rho}(m), m \in \Lambda_{n}$, determine $\rho$ in the space uniquely. We remark as an immediate consequence of (4.6),

$$
\hat{\rho}(j n)=0, \quad \rho \in \varsigma_{n}^{d}, j \in \mathbf{Z}^{*} .
$$

(Recall that the asterisk denotes the complement of $\{0\}$ in a set of integers.)

Now let $\rho \in \varsigma_{n}^{d}$ be arbitrary. It follows from (4.6), (4.7), and the fact that every integer can be written in a unique way as $p+m n$ with $p \in \Lambda_{n}, m \in \mathbf{Z}$, that

$$
\begin{aligned}
\|\rho\|_{s, \boldsymbol{E}}^{2}= & \sum_{p \in \Lambda_{n}} \sum_{m \in \mathbf{Z}}|\hat{\rho}(p+m n)|^{2} \varepsilon^{2 p+m n \mid}(\underline{p+m n})^{2 s} \\
= & |\hat{\rho}(0)|^{2}+(2 \pi)^{2 s} \sum_{p \in \Lambda_{n}^{*}}|\hat{\rho}(p)|^{2} p^{2 d+2} \sum_{m \in \mathbf{Z}} \varepsilon^{2 p+m n \mid}|p+m n|^{2 s-2 d-2} \\
= & |\hat{\rho}(0)|^{2}+\sum_{p \in \Lambda_{n}^{*}}|\hat{\rho}(p)|^{2} \varepsilon^{2|p|} \underline{p}^{2 s}\left|2 p n^{-1}\right|^{2 d+2-2 s} \\
& \times \sum_{m \in \mathbf{Z}} \varepsilon^{2 p+m n|-2| p \mid}\left|2 p n^{-1}+2 m\right|^{2 s-2 d-2}
\end{aligned}
$$

Now for $p \in \Lambda_{n}, m \in \mathbf{Z}, \varepsilon \in(0,1]$ we have $\left|2 p n^{-1}\right|<1$ and $|p+m n|-|p| \geqslant 0$. By hypothesis $r:=2 s-2 d-2 \leqslant 2 s_{0}-2 d_{0}-2<-1$. Thus

$$
\begin{aligned}
& \sum_{m \in \mathbf{Z}} \varepsilon^{2|p+m n|-2|p|}\left|2 p n^{-1}+2 m\right|^{r} \\
& \leqslant\left|2 p n^{-1}\right| r+\sum_{m=1}^{\infty}\left(2 m+2 p n^{-1}\right)^{r}+\sum_{m=1}^{\infty}\left(2 m-2 p n^{-1}\right)^{r} \\
& \leqslant\left|2 p n^{-1}\right|^{r}+\sum_{m=1}^{\infty}(2 m)^{r}+\sum_{m=1}^{\infty}(2 m-1)^{r}=\left|2 p n^{-1}\right|^{r}+\zeta(-r),
\end{aligned}
$$

where $\zeta(-r)=\sum_{m=1}^{\infty} m^{r}$ is Riemann's zeta function. Note that

$$
\zeta(-r) \leqslant \zeta\left(2 d_{0}+2-2 s_{0}\right)<\infty .
$$


By substituting (4.9) in (4.8), we complete the proof of the lemma with $C_{2}=1+$ $\zeta\left(2 d_{0}+2-2 s_{0}\right)$.

Proof of Theorem 4.1. Given nonzero $\rho \in \mathcal{S}_{n}^{d}$ set

$$
\sigma(x)=\sum_{k \in \Lambda_{n}} \overline{\hat{\rho}(k)} \varepsilon^{2|k|} \underline{k}^{2 s+1} e^{2 \pi i k x} .
$$

Then $\sigma \in \mathcal{T}_{n}$ and, in light of Lemma 4.2, $\rho \neq 0$ implies $\sigma \neq 0$. By the lemma, Parseval's identity, and (4.4),

$$
\|\rho\|_{s, \varepsilon}^{2} \leqslant C_{2} \sum_{k \in \Lambda_{n}}|\hat{\rho}(k)|^{2} \varepsilon^{2|k|} \underline{k}^{2 s}=C_{2}(V \rho, \sigma) .
$$

Moreover, from (4.10) and (3.2),

$$
\|\sigma\|_{-s-1, \varepsilon^{-1}}^{2}=\sum_{k \in \Lambda_{n}}|\hat{\rho}(k)|^{2} \varepsilon^{2|k|} \underline{k}^{2 s}=(V \rho, \sigma) .
$$

Combining (4.11) and (4.12), we get the theorem.

Remark. There is no question of extending Theorem 4.1 to $\varepsilon>1$ or $\varepsilon=1$ and $s \geqslant d+1 / 2$, since for such $s$ and $\varepsilon, \delta_{n}^{d} \not \subset X_{s, \varepsilon}$. Restricting to the case $\varepsilon=1$, we have shown that the spline-trig Galerkin method is stable in every Sobolev space containing the spline trial space, in the case $\Gamma=$ unit circle, $x(\tau)=e^{2 \pi i \tau}$. In fact we shall see that this holds true in general (as long as $x$ is a $C^{\infty}$ parametrization).

We now examine the difference between the operators $A$ and $V$. We begin with some preliminary lemmas.

Lemma 4.3. The function $K: \mathbf{R}^{2} \rightarrow \mathbf{R}$ defined

$$
K(\tau, t)= \begin{cases}-\frac{1}{\pi} \log \left|\frac{x(\tau)-x(t)}{2 \sin \pi(\tau-t)}\right|, & \tau-t \notin \mathbf{Z}, \\ -\frac{1}{\pi} \log \left|\frac{x^{\prime}(\tau)}{2 \pi}\right|, & \tau-t \in \mathbf{Z},\end{cases}
$$

is real analytic and is 1-periodic in each variable. Moreover $K$ extends analytically to $S_{\delta} \times S_{\delta}$ for some $\delta>0$, where $S_{\delta}=\{z \in \mathbf{C}|| \operatorname{Im} z \mid<\delta\}$.

Proof. It is clear that the function $F: \mathbf{R}^{2} \rightarrow \mathbf{R}^{2}$ defined by

$$
F(\tau, t)= \begin{cases}\frac{x(\tau)-x(t)}{2 \sin \pi(\tau-t)}, & \tau-t \notin \mathbf{Z}, \\ \frac{x^{\prime}(\tau)}{2 \pi}, & \tau-t \in \mathbf{Z},\end{cases}
$$

is analytic. Since $\left.x\right|_{[0,1)}$ is univalent and $x^{\prime}$ is nonvanishing on $\mathbf{R}, F$ does not vanish. Since $\log |\cdot|: \mathbf{R}^{2} \backslash\{0\} \rightarrow \mathbf{R}$ is analytic, $K$ is also analytic. Clearly $K$ is 1 -periodic in each variable. Finally the last sentence of the lemma follows from the previous.

The following lemma, which is proved in [8, Section 2.1], will be used to bound the Fourier coefficients of $K$.

LEMMA 4.4. If $f$ is analytic on $\overline{S_{\delta}}=\{z \in \mathrm{C}|| \operatorname{Im} z \mid \leqslant \delta\}$ and 1-periodic, then

$$
|\hat{f}(m)| \leqslant e^{-2 \pi \delta|m|}\|f\|_{L^{\infty}\left(S_{\delta}\right)}, \quad m \in \mathbf{Z} \text {. }
$$


We are now prepared to bound the Fourier coefficients of $K$, defined by

$$
\hat{K}(p, q)=\int_{0}^{1} \int_{0}^{1} K(\tau, t) e^{-2 \pi i(p \tau+q t)} d \tau d t, \quad p, q \in \mathbf{Z} .
$$

The following lemma follows from Lemmas 4.3 and 4.4.

LeMma 4.5. There exist constants $C_{3}>0, \varepsilon_{1} \in(0,1)$ such that

$$
|\hat{K}(p, q)| \leqslant C_{3} \varepsilon_{1}^{|p|+|q|}, \quad p, q \in \mathbf{Z} .
$$

Now from (2.3), (4.1), and (4.2),

$$
B \varphi(\tau):=A \varphi(\tau)-V \varphi(\tau)=\int_{0}^{1} K(\tau, t)[\varphi(t)-\hat{\varphi}(0)] d t
$$

We shall now use the decay estimates of the preceding lemma to prove that $B$ is a compact operator $X_{s, \varepsilon} \rightarrow X_{s+1, \varepsilon}$.

THEOREM 4.6. For $\varepsilon \in\left(\varepsilon_{1}, 1\right]$ ( $\varepsilon_{1}$ as in Lemma 4.5) and $t \in \mathbf{R}$, the operator $B$ extends boundedly to a map $X_{0, \varepsilon} \rightarrow H_{t}=X_{t, 1}$.

Proof. For $\varphi \in \mathcal{T}$ we have by Parseval's identity that

$$
B \varphi(\tau)=\sum_{q \in \mathbf{Z}^{*}} \hat{\varphi}(q) \int_{0}^{1} K(\tau, t) e^{2 \pi i q t} d t
$$

so

$$
\widehat{\widehat{B \varphi}(p)}=\sum_{q \in \mathbf{Z}^{*}} \hat{\varphi}(q) \overline{\hat{K}(p, q)}, \quad p \in \mathbf{Z} .
$$

Applying Lemma 4.5 and the Schwarz inequality, we have

$$
|\widehat{B \varphi}(p)|^{2} \leqslant C_{3}^{2}\left[\sum_{q}\left(\varepsilon_{1} / \varepsilon\right)^{2|q|}\right] \varepsilon_{1}^{2|p|} \sum_{q}|\hat{\varphi}(q)|^{2} \varepsilon^{2|q|},
$$

so

$$
\|B \varphi\|_{t}^{2}=\sum|\widehat{B \varphi}(p)|^{2} \underline{p}^{2 t} \leqslant C_{4}\|\varphi\|_{0, \varepsilon}^{2}
$$

where

$$
C_{4}=C_{3}^{2} \sum_{q}\left(\varepsilon_{1} / \varepsilon\right)^{2|q|} \sum_{p} \varepsilon_{1}^{2|p|} \underline{p}^{2 t}<\infty
$$

Corollary 4.7. For $\varepsilon \in\left(\varepsilon_{1}, 1\right], s \in \mathbf{R}, B$ maps $X_{s, \varepsilon}$ compactly into $X_{s+1, \varepsilon}$.

Proof. Choose $\varepsilon^{\prime} \in\left(\varepsilon_{1}, \varepsilon\right)$. Then $X_{s, \varepsilon}$ is compactly included in $X_{0, \varepsilon^{\prime}}, B$ maps $X_{0, \varepsilon^{\prime}}$ boundedly into $X_{s+1,1}$, and $X_{s+1,1}$ is contained in $X_{s+1, \varepsilon}$.

Corollary 4.8. For $\varepsilon \in\left(\varepsilon_{1}, 1\right], s \in \mathbf{R}, A$ maps $X_{s, \varepsilon}$ isomorphically onto $X_{s+1, \varepsilon}$.

Proof. Since $V$ is an isomorphism between the spaces in question and, by Corollary 4.7, $A$ is a compact perturbation of $V, A$ is a Fredholm mapping of index 0 . Thus it suffices to show that if $A \rho=0$ for some $\rho \in X_{s, \varepsilon}$, then $\rho=0$. Now for such $\rho$ Theorem 4.6 implies $V \rho=-B \rho \in H^{t}$ for all $t$, whence $\rho$ is a smooth function. Set $\omega=\hat{\rho}(0)$ and define a function $\psi$ on $\Gamma$ of mean value zero by $\psi(x(t))=$ $2[\rho(t)-\omega] /\left|x^{\prime}(t)\right|$. Since $A \rho=0$, the integral equation (1.3) is satisfied with $\tilde{g}=0$. By [9], [11], [14], however, this equation is uniquely solvable, so $\psi=0$ and $\omega=0$, whence $\rho=0$. 
We have shown that $A$ is a compact perturbation of $V$ and that the projection associated with $V$ is stable in the sense of Theorem 4.1. For the convenience of the reader we recall the theorem [3, Lemma 4.2] that allows us to conclude stability of the projection associated with $A$.

THEOREM 4.9. Let $Y$ and $Z$ be Banach spaces, $\mathbb{Q}: Y \rightarrow Z$ a linear isomorphism, $\mathfrak{H}:$ $Y \rightarrow Z$ a compact linear operator. For each $m$ in some directed set let $\mathfrak{N}_{m}$ and $\mathfrak{X}_{m}$ be given finite-dimensional subspaces of $Y$ and $Z^{\prime}$ (the dual space to $Z$ ), respectively. Suppose

(1) there exists $\gamma>0$ such that for all $m$ and $u \in \Re_{m}$ there exists nonzero $v \in \mathscr{N}_{m}$ satisfying

$$
\langle Q u, v\rangle \geqslant \gamma\|u\|_{Y}\|v\|_{Z^{\prime}}-\langle\mathscr{K} u, v\rangle
$$

(2) for all $z \in Z^{\prime}, \lim _{m} \operatorname{dist}\left(z, \Re_{m}\right)=0$.

Then there exists $\beta>0$ such that for all sufficiently large $n$

$$
\inf _{0 \neq u \in \Re_{m}} \sup _{0 \neq v \in \Re_{n}} \frac{\langle\mathfrak{Q} u, v\rangle}{\|u\|_{Y}\|v\|_{Z^{\prime}}} \geqslant \beta .
$$

From this theorem together with Corollary 4.7, Corollary 4.8, and Theorem 4.1, we may infer the stability of the spline-trig method. Invoking the standard theory of variational projections [4], [5, Chapter 5] we have the main theorem of this section.

THEOREM 4.10. There exists an integer $N$ depending only on $d$ such that for $n \geqslant N$ and $g \in X$ the discrete equations (2.4) of the spline-trig method have a unique solution $\varphi_{n} \in \mathcal{S}_{n}^{d}$. For $s \in(-\infty, d+1 / 2)$ and $\varepsilon \in\left(\varepsilon_{1}, 1\right]$ there exists a constant $C_{5}$ depending on $s, \varepsilon$, and $d$ such that, for $g \in X_{s+1, \varepsilon}$ and $n \geqslant N$,

$$
\left\|\varphi-\varphi_{n}\right\|_{s, \varepsilon} \leqslant C_{5} \inf _{\rho \in \mathcal{S}_{n}^{d}}\|\varphi-\rho\|_{s, \varepsilon} .
$$

Remark 4.11. We used the analyticity of the parametrization $x$ to obtain the compactness result of Corollary 4.7. If $x$ were merely $C^{\infty}$, we could still establish the result in case $\varepsilon=1$ by a similar argument, using the fact that the Fourier coefficients of a $C^{\infty}$ function decay faster than any polynomial. The stability result thus would still hold for the Sobolev spaces $(\varepsilon=1)$. If $x$ had only a finite number of derivatives, this result would hold only for an appropriately restricted range of the index $s$.

5. Convergence Analysis. For $\varphi \in X$ let $P_{n} \varphi \in \delta_{n}^{d}$ be defined by

$$
\left(V P_{n} \varphi, \sigma\right)=(V \varphi, \sigma), \quad \sigma \in \mathcal{T}_{n},
$$

or, equivalently,

$$
\widehat{P_{n} \varphi}(k)=\hat{\varphi}(k), \quad k \in \Lambda_{n} .
$$

From (4.5) and Theorem 4.1 we see that $P_{n}$ is well defined for each $n$ and that $P_{n} \varphi$ provides quasioptimal approximation to $\varphi$ in all the spaces $X_{s, \varepsilon}, s<d+1 / 2$, $\varepsilon \in(0,1]$. We now determine the asymptotic rates of convergence, which by Theorem 4.10 will also give bounds on the error $\varphi-\varphi_{n}$ in the spline-trig method.

TheOrem 5.1. Let $s \in(-\infty, d+1 / 2), \varepsilon \in(0,1]$, and $t \in[s, d+1]$. Then

$$
\left\|\varphi-P_{n} \varphi\right\|_{s, \varepsilon} \leqslant \sqrt{2 \zeta(2 d+2-2 s)} \varepsilon^{n / 2}(\pi n)^{s-t}\|\varphi-\hat{\varphi}(0)\|_{t}, \quad \varphi \in H^{t} .
$$


(Here $\zeta$ again denotes the Riemann zeta function. Note that if $s \leqslant s_{0}<d+1 / 2$, then $\zeta(2 d+2-2 s) \leqslant \zeta\left(2 d+2-2 s_{0}\right)<\infty$, and in particular $\zeta(2 d+2-2 s) \leqslant$ $\pi^{2} / 6$ for $s \leqslant d$. However $\lim _{s \uparrow d+1 / 2} \zeta(2 d+2-2 s)=+\infty$.)

Proof. From (5.1) and (3.2)

$$
\left\|\varphi-P_{n} \varphi\right\|_{s, \varepsilon}^{2} \leqslant 2 \sum_{k \notin \Lambda_{n}}\left[|\hat{\varphi}(k)|^{2}+\left|\widehat{P_{n} \varphi}(k)\right|^{2}\right] \varepsilon^{2|k|} \underline{k}^{2 s} .
$$

Now for $k \in \mathbf{Z} \backslash \Lambda_{n}, 2|k| \geqslant n$, so

$$
\sum_{k \notin \Lambda_{n}}|\hat{\varphi}(k)|^{2} \varepsilon^{2|k|} \underline{k}^{2 s} \leqslant \varepsilon^{n}(\pi n)^{2 s-2 t} \sum_{k \notin \Lambda_{n}}|\hat{\varphi}(k)|^{2} \underline{k}^{2 t} .
$$

Applying (4.6), we also get

$$
\begin{aligned}
\sum_{k \notin \Lambda_{n}}\left|\widehat{P_{n} \varphi}(k)\right|^{2} \varepsilon^{2|k|} \underline{k}^{2 s} & \\
= & \sum_{p \in \Lambda_{n}^{*}}|\hat{\varphi}(p)|^{2} p^{2 d+2} \sum_{m \in \mathbf{Z}^{*}}|p+m n|^{-2 d-2} \varepsilon^{2|p+m n|}(\underline{p+m n})^{2 s} \\
= & (\pi n)^{2 s-2 t} \sum_{p \in \Lambda_{*}^{*}}|\hat{\varphi}(p)|^{2} \underline{p}^{2 t}\left|2 p n^{-1}\right|^{2 d+2-2 t} \\
& \times \sum_{m \in \mathbf{Z}^{*}}\left|2 p n^{-1}+2 m\right|^{-2 d-2+2 s} \varepsilon^{2|p+m n|} \\
\leqslant & \varepsilon^{n}(\pi n)^{2 s-2 t} \zeta(2 d+2-2 s) \sum_{p \in \Lambda_{n}^{*}}|\hat{\varphi}(p)|^{2} \underline{p}^{2 t} .
\end{aligned}
$$

The theorem follows directly from (5.2), (5.3), and (5.4).

From Theorems 4.10 and 5.1 we infer immediately the following optimal convergence estimates for the spline-trig method.

TheOREM 5.2. Let $s \in(-\infty, d+1 / 2), \varepsilon \in\left(\varepsilon_{1}, 1\right], t \in[s, d+1]$. Then there exists a constant $C_{6}$ such that if the solution $\varphi$ to (2.3) is in $H^{t}$ (i.e., if $g \in H^{t+1}$ ) and $\varphi_{n} \in \mathcal{S}_{n}^{d}$ is the spline-trig approximation defined by (2.4) for $n \geqslant N$, then

$$
\left\|\varphi-\varphi_{n}\right\|_{s, \varepsilon} \leqslant C_{6} \varepsilon^{n / 2} n^{s-t}\|\varphi-\hat{\varphi}(0)\|_{t} .
$$

We now analyze the approximation to the solution $u$ of (1.1) which may be derived from $\varphi_{n}$. Define

$$
u_{n}(z)=-\frac{1}{\pi} \int_{0}^{1} \log \mid z-x(t) \|\left[\varphi_{n}(t)-\hat{\varphi}_{n}(0)\right] d t+\hat{\varphi}_{n}(0), \quad z \in \mathbf{R}^{2}
$$

We may also compute an approximation to $\partial^{\alpha} u(z)$, the partial derivative of $u$ with respect to $z$ for any nonzero multi-index $\alpha \in \mathbf{N}^{2}$, by a quadrature:

$$
\partial^{\alpha} u_{n}(z)=-\frac{1}{\pi} \int_{0}^{1} \partial^{\alpha} \log |z-x(t)|\left[\varphi_{n}(t)-\hat{\varphi}_{n}(0)\right] d t, \quad z \in \mathbf{R}^{2} \backslash \Gamma .
$$

THEOREM 5.3. Let $\Omega$ be a compact subset of $\mathbf{R}^{2} \backslash \Gamma, \alpha \in \mathbf{N}^{2}, t \in(\infty, d+1]$. Then there exist constants $C_{7} \in(0, \infty)$ and $\varepsilon_{2} \in(0,1)$ such that if $\varphi \in H^{t}$, then for $n$ sufficiently large

$$
\left\|\partial^{\alpha}\left(u-u_{n}\right)\right\|_{L^{\infty}(\Omega)} \leqslant C_{7} \varepsilon_{2}^{n}\|\varphi-\hat{\varphi}(0)\|_{t}
$$


Proof. It suffices to prove the theorem in the case $\Omega$ is a closed disc in $\mathbf{R}^{2} \backslash \Gamma$. By (2.2) and (5.5) we have

$$
\begin{aligned}
u(z)-u_{n}(z)= & -\frac{1}{\pi} \int_{0}^{1} \log |z-x(t)|\left[\varphi(t)-\varphi_{n}(t)-\hat{\varphi}(0)+\hat{\varphi}_{n}(0)\right] d t \\
& +\hat{\varphi}(0)-\hat{\varphi}_{n}(0), \quad z \in \mathbf{R}^{2} .
\end{aligned}
$$

Since $z-x(t)$ does not vanish for $(t, z) \in \mathbf{R} \times \Omega, L(t, z)=\log |z-x(t)|$ is an analytic function of three real variables on $\mathbf{R} \times \Omega$. Since $L$ is periodic with respect to $t$, it extends to a holomorphic function $\overline{S_{\delta}} \times \Omega \rightarrow \mathrm{C}$ for some $\delta>0$, where $\overline{S_{\delta}}=$ $\{w \in \mathbf{C}|| \operatorname{Im} w \mid \leqslant \delta\}$. By compactness, $\sup \left\{|L(t, z)| \mid t \in \bar{S}_{\delta}, z \in \Omega\right\}<\infty$. Setting $L_{z}(t)=L(t, z)$, we may apply Lemma 4.4 to get

$$
\left|\hat{L}_{z}(m)\right| \leqslant C_{8} \varepsilon_{3}^{|m|}, \quad m \in \mathbf{Z}, z \in \Omega,
$$

for constants $C_{8}>0, \varepsilon_{3} \in(0,1)$.

Take $\eta \in\left(\max \left(\varepsilon_{1}, \varepsilon_{3}\right), 1\right)$, and set $\varepsilon_{2}=\sqrt{\eta}$. We then have

$$
\sup _{z \in \Omega}\left\|L_{z}\right\|_{-t, \eta^{-1}}<\infty
$$

whence, by Theorem 5.2,

$$
\begin{aligned}
& \left|\int_{0}^{1} \log \right| z-x(t)\left|\left[\varphi(t)-\varphi_{n}(t)\right] d t\right| \\
& \leqslant\left\|L_{z}\right\|_{-t, \eta^{-1}}\left\|\varphi-\varphi_{n}\right\|_{t, \eta} \leqslant C_{9} \varepsilon_{2}^{n}\|\varphi-\hat{\varphi}(0)\|_{t},
\end{aligned}
$$

where $C_{9}$ depends only on $\Omega$ and $t$. Since also

$$
\left|\hat{\varphi}(0)-\hat{\varphi}_{n}(0)\right| \leqslant\left\|\varphi-\varphi_{n}\right\|_{t, \eta},
$$

the theorem is proved in the case $\alpha=0$. Since

$$
\begin{aligned}
& \partial^{\alpha} u(z)-\partial^{\alpha} u_{n}(z) \\
& =-\frac{1}{\pi} \int_{0}^{1} \partial^{\alpha} \log |z-x(t)|\left[\varphi(t)-\varphi_{n}(t)-\hat{\varphi}(0)+\hat{\varphi}_{n}(0)\right] d t, \\
& \qquad z \in \mathbf{R}^{2} \backslash \Gamma,
\end{aligned}
$$

an altogether similar argument suffices to prove the theorem for nonzero $\alpha$.

We have thus shown exponential convergence to the solution of the Dirichlet problem and all its derivatives away from the boundary. We conclude our convergence analysis by giving polynomial convergence rates which are valid up to the boundary for $u_{n}$ and its derivatives of order up to $d+1$ measured in $L^{\infty}$ and $L^{2}$.

THEOREM 5.4. Let $\alpha \in \mathbf{N}^{2}$ be a multi-index of degree $k=\alpha_{1}+\alpha_{2} \leqslant d+1$, and let $t \in(k-1 / 2, d+1]$. Then there exists a constant $C_{10}$ such that if $\varphi \in H^{t}$ and $n \geqslant N$,

$$
\left\|\partial^{\alpha}\left(u-u_{n}\right)\right\|_{L^{\infty}\left(\mathbf{R}^{2} \backslash \Gamma\right)} \leqslant C_{10} n^{k-t-1 / 2} \sqrt{\log n}\|\varphi-\hat{\varphi}(0)\|_{t} .
$$

Proof. From (2.2) and (5.5) we see that both $u$ and $u_{n}$ are harmonic functions on $\mathbf{R}^{2} \backslash \Gamma$ and that both are harmonic (i.e., bounded) at infinity (the integral terms in (2.2) and (5.5) both tend to zero as $|z|$ tends to infinity). It follows, as can easily be verified with help of the Kelvin transform, that for all $\alpha, \partial^{\alpha} u$ and $\partial^{\alpha} u_{n}$ are also harmonic on $\left(\mathbf{R}^{2} \backslash \Gamma\right) \cup\{\infty\}$. Thus we may apply the maximum principle in both the interior and exterior domains. Letting $e_{+}^{\alpha}$ and $e_{-}^{\alpha}$ denote the traces on $\Gamma$ of 
$\partial^{\alpha}\left(u-u_{n}\right)$ from the interior and exterior domains, respectively (for $|\alpha|>0$ these will not coincide), we see that it suffices to bound these two functions in $L^{\infty}(\Gamma)$ by the quantity on the right-hand side of (5.8). Let $B_{+}$denote the interior domain and

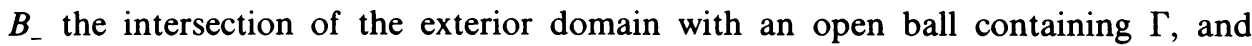
denote by \pm either the symbol + or - . Using a standard trace inequality, we have for $s>0$ that

$$
\left\|e_{ \pm}^{\alpha} \circ x\right\|_{s}=\left\|e_{ \pm}^{\alpha}\right\|_{H^{s}(\Gamma)} \leqslant C_{11}\left\|\partial^{\alpha}\left(u-u_{n}\right)\right\|_{H^{s+1 / 2\left(B_{ \pm}\right)}} \leqslant C_{12}\left\|u-u_{n}\right\|_{H^{s+k+1 / 2}\left(B_{ \pm}\right)} .
$$

Using well-known regularity theory for the Laplacian in the interior domain together with the Kelvin transform in the case of the exterior domain and then applying Corollary 4.8 , we have

$$
\left\|e_{ \pm}^{\alpha} \circ x\right\|_{s} \leqslant C_{13}\left\|u-u_{n}\right\|_{H^{s+k}(\Gamma)} \leqslant C_{14}\left\|\varphi-\varphi_{n}\right\|_{s+k-1} .
$$

Now, from the Fourier expansion (3.1) and the definition (3.2) of the norm in $H^{s}$, we easily establish the Sobolev inequality

$$
\|f\|_{L^{\infty}(\mathbf{R})} \leqslant C(\mu)\|f\|_{1 / 2+\mu}, \quad \mu>0, f \in H^{1 / 2+\mu},
$$

where $C(\mu)^{2}=\Sigma_{k \in \mathbf{Z}} \underline{k}^{-1-2 \mu}=1+2(2 \pi)^{-1-2 \mu} \zeta(1+2 \mu)$. Since the zeta function has a simple pole at 1 , it follows that $C(\mu) \leqslant C_{15} \mu^{-1 / 2}$ for $0<\mu \leqslant 1$. Combining (5.10), (5.9), and Theorem 5.2, we get

$$
\left\|e_{ \pm}^{\alpha}\right\|_{L^{\infty}(\Gamma)} \leqslant C_{6} C_{14} C_{15} \mu^{-1 / 2} n^{\mu+k-t-1 / 2}\|\varphi-\hat{\varphi}(0)\|_{t} .
$$

Setting $\mu=(\log n)^{-1}$, the right-hand side becomes

$$
e C_{6} C_{14} C_{15} n^{k-t-1 / 2} \sqrt{\log n}\|\varphi-\hat{\varphi}(0)\|_{t}
$$

as desired.

Measured in $L^{2}$ on a bounded subset of $\mathbf{R}^{2}$ the derivatives $\partial^{\alpha}\left(u-u_{n}\right)$ of the error actually converge with higher order than indicated in Theorem 5.4, as we now prove. To this end we require in addition to the usual Sobolev spaces $H^{k}\left(\Omega_{+}\right)$on the interior domain $\Omega_{+}$also certain weighted Sobolev spaces $W^{k}\left(\Omega_{-}\right)$on the exterior domain $\Omega_{-}$. Following [14] we define $W^{k}\left(\Omega_{-}\right)$for $k \in \mathbf{N}$ as the set of distributions $v$ in $\Omega_{-}$for which the following norm is finite:

$$
\|v\|_{W^{k}\left(\Omega_{-}\right)}=\left\{\int_{\Omega_{-}}\left[\frac{|u(z)|^{2}}{\left(1+r^{2}\right)\left(1+\log \sqrt{1+r^{2}}\right)^{2}}+\sum_{1 \leqslant|\alpha| \leqslant k} \frac{\left|\partial^{\alpha} u(z)\right|^{2}}{\left(1+r^{2}\right)^{1-|\alpha|}}\right] d z\right\}^{1 / 2},
$$

where $r=|z|$.

THEOREM 5.5. Let $k \leqslant d+1$ be a positive integer and $t \in[k-3 / 2, d+1]$. Then there exists a constant $C_{16}$ such that if $\varphi \in H^{t}$ and $n \geqslant N$, then

$$
\left\|u-u_{n}\right\|_{H^{k}\left(\Omega_{+}\right)}+\left\|u-u_{n}\right\|_{W^{k}\left(\Omega_{-}\right)} \leqslant C_{16} n^{k-t-3 / 2}\|\varphi-\hat{\varphi}(0)\|_{t} .
$$

Proof. It follows directly from [14, Theorems 1.1 and 1.3] that the left-hand side of (5.11) is bounded by a multiple of $\left\|\varphi-\varphi_{n}\right\|_{k-3 / 2}$, so Theorem 5.5 is a consequence of Theorem 5.2.

Remark 5.6. Since the stability result of Theorem 4.10 holds in the case $\varepsilon=1$ if the parametrization $x$ is only $C^{\infty}$ (Remark 4.11), the same applies to the convergence 
result of Theorem 5.2. Since the proofs of Theorems 5.4 and 5.5, concerning convergence up to the boundary, require only the case $\varepsilon=1$ of Theorem 5.2 , these theorems also hold for a $C^{\infty}$ parametrization. A modification to the proof of Theorem 5.3 shows that, in this more general case, $u-u_{n}$ and its derivatives tend to zero uniformly on compact sets disjoint from $\Gamma$ faster than any polynomial in $1 / n$, although we require the analyticity of $x$ to obtain the exponential rate.

6. Conditioning. It is worth pointing out that the stiffness matrix associated with our method has an $l_{2}$ condition number that grows only with first order as $n$ increases. This is the same order as is achieved by an ordinary spline Galerkin method.

Let

$$
\left\{\omega_{1}, \ldots, \omega_{n}\right\}=\left\{\chi_{n}^{d+1}(\cdot-j / n) \mid j \in \Lambda_{n}\right\}
$$

and

$$
\left\{\nu_{1}, \ldots, \nu_{n}\right\}=\{1, \sqrt{2} \sin (2 \pi \cdot), \ldots, \sqrt{2} \cos ((n-1) \pi \cdot)\}
$$

be bases of $\mathcal{S}_{n}^{d}$ and $\mathcal{T}_{n}$, respectively. Then the stiffness matrix $M \in \mathbf{R}^{n \times n}$ has $(j, k)$ entry $\left(A \omega_{k}, \nu_{j}\right)$. Now let $\alpha \in \mathbf{R}^{n}$ be arbitrary. We shall show that

$$
|M \alpha| \leqslant \bar{\lambda}_{n}\|A\|_{e\left(L^{2}, L^{2}\right)}|\alpha|
$$

and

$$
|\alpha| \leqslant \underline{\lambda}_{n}^{-1} C_{n} n \pi\left\|A^{-1}\right\|_{\mathcal{L}\left(H^{1}, L^{2}\right)}|M \alpha|,
$$

where $|\cdot|$ denotes the usual Euclidean norm on $\mathbf{R}^{n}, C_{n}$ denotes the $L^{2}$ operator norm of the spline-trig projectior $\varphi \mapsto \varphi_{n}$, and $\bar{\lambda}_{n}$ and $\underline{\lambda}_{n}$ denote the supremum and infimum, respectively, over $\mathbf{R}^{n} \backslash\{0\}$ of the ratio $\left\|\sum \alpha_{j} \omega_{j}\right\|_{0} /|\alpha|$. Now $A$ is an isomorphism of $L^{2}$ onto $H^{1}$ (Corollary 4.8), and $C_{n}$ is bounded independent of $n$ (Theorem 4.10). Since the ratio $\bar{\lambda}_{n} / \underline{\lambda}_{n}$ is bounded independent of $n$ [15, Lemma 14], (6.1) and (6.2) indeed show that the $l_{2}$ condition number of $M$ grows with at most first order in $n$.

Let $\beta=M \alpha \in \mathbf{R}^{n}, f=\sum_{j=1}^{n} \beta_{j} \nu_{j} \in \mathcal{T}_{n}, \varphi=A^{-1} f$, and let $\varphi_{n}$ be the spline-trig approximation of $\varphi$. Then it is easily verified that $\varphi_{n}=\sum_{k=1}^{n} \alpha_{k} \omega_{k}$. Therefore $f$ is the $L^{2}$ projection of $A \varphi_{n}$ on $\mathcal{T}_{n}$ and

$$
|\beta|=\|f\|_{0} \leqslant\left\|A \varphi_{n}\right\|_{0} \leqslant\|A\|_{\mathcal{L}\left(L^{2}, L^{2}\right)}\left\|\varphi_{n}\right\|_{0} .
$$

Noting that $\left\|\varphi_{n}\right\|_{0} \leqslant \bar{\lambda}_{n}|\alpha|$, this demonstrates (6.1). For (6.2) we note first that

$$
|\alpha| \leqslant \underline{\lambda}_{n}^{-1}\left\|\varphi_{n}\right\|_{0} \leqslant \underline{\lambda}_{n}^{-1} C_{n}\|\varphi\|_{0} \leqslant \underline{\lambda}_{n}^{-1} C_{n}\left\|A^{-1}\right\|_{\mathcal{L}\left(H^{1}, L^{2}\right)}\|f\|_{1} .
$$

Since $\|f\|_{1} \leqslant n \pi\|f\|_{0}$ for $f \in \mathcal{T}_{n},(6.2)$ is proved.

Department of Mathematics

University of Maryland

College Park, Maryland 20742

1. D. Arnold, I. Babuska \& J. Osborn, "Selection of finite element methods," Proc. Internat. Sympos. on Hybrid and Mixed Finite Element Methods (S. N. Atluri and R. H. Gallagher, eds.), Wiley, New York, 1981.

2. D. ARnold \& W. Wendland, "On the asymptotic convergence of collocation methods," Math. Comp.. v. 41, 1983, pp. 
3. A. Aziz \& B. Kellogg, "Finite element analysis of a scattering problem." Math. Comp., v. 37. 1981, pp. 261-272.

4. I. BABUSKa, “Error-bounds for finite element method,” Numer. Math., v. 16, 1970, pp. 322-333.

5. I. BABUSKA \& A. K. AzIZ, "Survey lectures on the mathematical foundations of the finite element method," The Mathematical Foundations of the Finite Element Method with Applications to Partial Differential Equations (A. K. Aziz, ed.), Academic Press, New York, 1972.

6. G. FicherA, "Linear elliptic equations of higher order in two independent variables and singular integral equations," Proc. Conf. on Partial Differential Equations and Continuum Mechanics (Madison, Wisc.), Univ. of Wisconsin Press, 1961.

7. H.-P. HelfRICH, "Simultaneous approximation in negative norms of arbitrary order," RAIRO Anal. Numer., v. 15, 1981, pp. 231-235.

8. P. Henrici, "Fast Fourier methods in computational complex analysis," SIAM Rev., v. 15, 1979, pp. 481-527.

9. G. Hsiao, On the Stability of Integral Equations of the First Kind with Logarithmic Kernels, Tech. Rep. No. 103-A, Appl. Math. Inst., Univ. of Delaware, 1981.

10. G. HsiaO, P. KoPP \& W. WENDLAND, “A Galerkin collocation method for some integral equations of the first kind," Computing, v. 25, 1980, pp. 89-130.

11. G. HsiaO \& R. MCCAMY, "Solution of boundary value problems by integral equations of the first kind,” SIAM Rev., v. 15, 1973, pp. 687-705.

12. G. Hsiao \& W. Wendland, “A finite element method for some integral equations of the first kind,” J. Math. Anal. Appl., v. 58, 1977, pp. 449-481.

13. G. Hsiao \& W. Wendland, "The Aubin-Nitsche lemma for integral equations," J. Integral Equations, v. 3, 1981, pp. 299-315.

14. M. N. Le Roux, "Méthode d'éléments finis pour la résolution numérique de problèmes extérieurs en dimension 2," RAIRO Anal. Numér., v. 11, 1977, pp. 27-60.

15. I. Schoenberg, "Cardinal interpolation and spline functions," J. Approx. Theory, v. 2, 1969, pp. 167-206.

16. L. Schumaker, Spline Functions: Basic Theory, Wiley, New York, 1981.

17. G. Strang \& G. Fix, An Analysis of the Finite Element Method, Prentice-Hall, Englewood Cliffs, N.J., 1973. 